\title{
Differential Graded Lie Algebras and Singularities of Level Sets of Momentum Mappings
}

\author{
William M. Goldman and John J. Millson \\ Department of Mathematics, University of Maryland, College Park, Maryland 20742, USA \\ Dedicated to the memory of Bruce Reinhart
}

\begin{abstract}
The germ of an analytic variety $X$ at a point $x \in X$ is said to be quadratic if it is bi-analytically isomorphic to the germ of a cone defined by a system of homogeneous quadratic equations at the origin. Arms, Marsden and Moncrief show in [2] that under certain conditions the analytic germ of a level set of a momentum mapping is quadratic. We discuss related ideas in a more algebraic context by associating to an affine Hamiltonian action a differential graded Lie algebra, which in the presence of an invariant positive complex structure, is formal in the sense of [5].
\end{abstract}

\section{Introduction}

Let $V$ be a symplectic manifold and let a denote a Lie algebra. A Hamiltonian action of $\mathfrak{a}$ on $V$ consists of a Lie algebra homomorphism $\phi: \mathfrak{a} \rightarrow C^{\infty}(V)$, where $C^{\infty}(V)$ is the Lie algebra of smooth functions on $V$ under Poisson bracket. Associated with a Hamiltonian action is a momentum map $\Phi: V \rightarrow \mathfrak{a}^{*}$ defined by $\Phi(x): \xi \rightarrow \phi(\xi)(x)$, where $\mathfrak{a}^{*}$ is the vector space dual to $\mathfrak{a}$. The momentum map $\Phi$ is equivariant with respect to the action of $a$ on $V$ and the coadjoint action of $a$ on $a^{*}$. In [2], Arms, Marsden and Moncrief prove that under certain conditions, the analytic germ of the level set $\Phi^{-1}(0)$ at a fixed point $x_{0} \in \Phi^{-1}(0)$ is isomorphic to the analytic germ of a quadratic cone at the origin. In this paper we derive a related result by algebraic considerations in deformation theory, using a point of view we learned from P. Deligne [5] and elaborated in our papers [7,8]. Our main results are the following:

Theorem A. Let $A$ be a Lie group with Lie algebra a and $(E, \omega)$ be a finite dimensional symplectic affine space. Suppose $\alpha: A \rightarrow \operatorname{Aff}(E)$ is an affine Hamiltonian action with an equivariant momentum mapping $\Phi: E \rightarrow \mathfrak{a}^{*}$. Suppose that $\alpha$ preserves a parallel

\footnotetext{
* The first author was supported in part by the University of Maryland Institute for Advanced Computer Studies, National Science Foundation grant DMS-86-13576 and an Alfred P. Sloan Foundation Fellowship; the second by National Science Foundation grant DMS-85-01742
} 
positive definite complex structure $\mathbf{J}$ on $E$. If $x \in \Phi^{-1}(0)$, then the analytic germ of $\Phi^{-1}(0)$ at $x$ is isomorphic to a quadratic cone.

The quadratic nature of $\Phi^{-1}(0)$ in the presence of an invariant positive definite complex structure remains true in certain infinite dimensional situations as well. In this case one wants the result that the singularities of $\Phi^{-1}(0)$ look like the product of a finite dimensional quadratic cone with a closed affine subspace. Unfortunately it seems difficult to formulate an infinite dimensional version of Theorem A that will cover a reasonable number of interesting cases. We restrict ourselves to a statement including the gauge theory example following the statement of the theorem. In what follows the symbol $U^{\perp}$ for $U \subset T_{x}(E)$ denotes the orthogonal complement of $U$ for the symmetric form (, ) defined by

$$
(x, y)=\omega(x, \mathbf{J} y) \text {. }
$$

A weak symplectic form on a Banach manifold $V$ is a closed 2-form $\omega$ such that for each $x \in V$ the associated map $T_{x}(V) \rightarrow T_{x}(V)^{*}$ is injective (but possibly not surjective). An almost complex structure $\mathbf{J}$ is positive definite if the symmetric form $($,$) defined above is positive definite.$

If $\alpha: A \rightarrow \operatorname{Aff}(E)$ is an affine representation of a Lie group $A$ on an affine space $E$ and $A$ has Lie algebra a, we denote the associated representation of Lie algebras by $\bar{\alpha}: \mathfrak{a} \rightarrow \mathfrak{a f f f}(E)$.

Theorem B. Let $A$ be a Banach Lie group with Lie algebra a and $(E, \omega)$ be a weak symplectic affine space whose underlying vector space $V$ of translations is a Banach space. Suppose $\alpha: A \rightarrow \operatorname{Aff}(E)$ is an affine Hamiltonian action with an equivariant momentum mapping $\Phi: E \rightarrow \mathfrak{a}^{*}$. Suppose that $\alpha$ preserves a parallel positive definite complex structure $\mathbf{J}$ on $E$. Assume that $x \in \Phi^{-1}(0) \subset E$ satisfies the following conditions:

1. The evaluation map

$$
\begin{aligned}
e_{x}: \mathfrak{a} & \rightarrow T_{x} E \\
\lambda & \mapsto \bar{\alpha}(\lambda)(x)
\end{aligned}
$$

is continuous and its kernel has finite dimension;

2. The image $B^{1}=\operatorname{Image}\left(e_{x}: \mathfrak{a} \rightarrow T_{x} E\right)$ has finite codimension in $Z^{1}=\operatorname{Ker}\left(d \Phi: T_{x} E \rightarrow\right.$ $\left.T_{0} \mathfrak{a}^{*}\right)$

3. The differential of $\Phi: E \rightarrow \mathfrak{a}^{*}$ is continuous and its image has finite codimension; 4. $T_{x}(E)=B^{1}+\left(B^{1}\right)^{\perp}$, where $\left(B^{1}\right)^{\perp}$ is the orthogonal complement with respect to the weak metric $($,$) defined above.$

Then the germ of $\Phi^{-1}(0)$ at $x$ is Banach analytically isomorphic to the product germ $\left(B^{1}, 0\right) \times(\mathscr{Q}, 0)$, where $\mathscr{Q}$ is a finite dimensional quadratic cone.

The "Hodge decomposition"

$$
T_{x}(E)=B^{1}+\left(B^{1}\right)^{\perp}
$$

implies (via the inverse function theorem in Banach spaces) that there exists a submanifold $\mathscr{U}_{e}$ of $A$ containing the identity $e$ and a submanifold $S_{x}=\exp _{x}\left(B^{1}\right)^{\perp}$ 
of $E$ such that the map $\mu: \mathscr{U}_{e} \times S_{x} \rightarrow E$ given by $\mu(u, s)=\alpha(u) s$ is a diffeomorphism near $(e, x)$.

Since $d \Phi: T_{x} \rightarrow T_{0}$ a is a continuous linear map of Banach spaces with image of finite codimension, its image is closed. (For convenience of the reader we supply the proof: We may assume that $d \Phi$ is injective. Let $F$ be a finite dimensional subspace such that $F+\operatorname{Image}(d \Phi)=T_{0} \mathfrak{a}$ and $F \cap \operatorname{Image}(d \Phi)=0$. Then the natural map $F \oplus T_{x} E \rightarrow T_{0}$ a given by $(f, u) \mapsto f+d \Phi(u)$ is continuous and bijective; hence, by the open mapping theorem, it is a Banach isomorphism. Then Image $(d \Phi)$ is the image of the closed subspace $T_{x} E \subset F \oplus T_{x} E$ under this isomorphism.)

We observe that if $E$ is finite dimensional then $(*)$ holds because $($,$) is positive$ definite. However if $E$ is infinite dimensional it appears to be necessary to assume $(*)$ holds since $($,$) is only weakly non-degenerate. It is important to note that$ Theorems A and B remain true given only the germ of an action $A \times E \rightarrow E$ at $(e, x)$.

The starting point for this investigation was the observation [6] that for any compact Lie group $G$, the space of flat principal $G$-bundles over a compact Riemann surface has quadratic singularities in the above sense. This is related to the work of Arms-Marsden-Moncrief [2] and this relation is expounded in the appendix to [6]. In this case the symplectic affine space is the space $\mathscr{A}(P)$ of all smooth connections on a fixed principal $G$-bundle $P$ and the Lie algebra is the vector space $\Omega^{0}(M ;$ ad $P)$ of sections of the vector bundle ad $P$ associated to $P$ by the adjoint representation of $G$. An $\operatorname{Ad}(G)$-invariant nondegenerate inner product $\mathbf{B}$ on the Lie algebra of $G$ determines a pairing on the smooth ad $P$-valued differential forms

$$
\mathbf{B}_{*}(\cap[M]): \Omega^{i}(M ; \operatorname{ad} P) \times \Omega^{2-i}(M ; \operatorname{ad} P) \rightarrow \mathbb{R}
$$

by

$$
\mathbf{B}_{*}(\cap[M])(\alpha, \beta)=\int_{M} \mathbf{B}_{*}(\alpha \wedge \beta) .
$$

Since $\mathscr{A}(P)$ is an affine space with associated group of translations the vector space $\Omega^{1}(M ;$ ad $P)$, the above pairing defines a parallel symplectic structure on $\mathscr{A}(P)$. The gauge group $\mathscr{G}(P)$ consisting of bundle automorphisms $P \rightarrow P$ covering the identity map of $M$ acts affinely on $\mathscr{A}(P)$ and its Lie algebra is $\Omega^{0}(M ; \operatorname{ad} P)$. Furthermore this pairing gives an identification of a suitable Sobolev completion of $\Omega^{2}(M ; \operatorname{ad} P)$ with the coadjoint module of a suitable Sobolev completion of $\Omega^{\circ}(M ; \operatorname{ad} P)$ and, as first noted by Atiyah-Bott [3, p. 587], the function

$$
\Phi: \mathscr{A}(P) \rightarrow \Omega^{2}(M ; \text { ad } P) \subset \Omega^{0}(M ; \operatorname{ad} P)^{*}
$$

assigning to a connection its curvature is the momentum map for the action of $\mathscr{G}(P)$. The level set $\Phi^{-1}(0)$ in this case consists of the flat connections on $P$ and by [2] this space has quadratic singularities. In fact to apply Theorem B one must take a suitable Sobolev completion, see [3]. On the completion the form $\mathbf{B}_{*}$ is only weakly non-degenerate. The decomposition $(*)$ is the usual Hodge decomposition.

Since the material in [8] is basic to what follows we briefly summarize its contents. In [8] we construct a function $L \mapsto R_{L}$ from the category of differential 
graded Lie algebras $L$ over $\mathbb{R}$ with $H^{1}(L)$ finite-dimensional to the category $\hat{\mathfrak{U}}$ of complete local Noetherian $\mathbb{R}$-algebras as follows.

Choose a complement $C^{1}$ to the 1-boundaries $B^{1} \subset L^{1}$. Let $Y_{L}: \mathfrak{U} \rightarrow \hat{\mathfrak{Q}}$ be the functor defined on the category $\mathfrak{U}$ of Artin local $\mathbb{R}$-algebras given by:

$$
Y_{L}(A)=\left\{\omega \in C^{1} \otimes \mathrm{m}: d \omega+\frac{1}{2}[\omega, \omega]=0\right\} .
$$

Here $A$ is an object of $\mathfrak{A}$ and $\mathfrak{m}$ is its maximal ideal. It is easily verified that $Y_{L}$ satisfies the axioms of Schlessinger [9] and consequently there exists a complete local $\mathbb{R}$-algebra $R_{L}$ which pro-represents $Y_{L}$; that is, there is a natural isomorphism of functors on $\mathfrak{A}$,

$$
\operatorname{Hom}_{\mathbb{R}-\text { alg }}\left(R_{L}, \cdot\right) \rightarrow Y_{L}(\cdot) \text {. }
$$

Developing ideas of Deligne [4], we prove in [8, Theorem 4.1] that a differential graded Lie algebra homomorphism $f: L_{1} \rightarrow L_{2}$ inducing an isomorphism of cohomology induces an isomorphism from $R_{L_{1}}$ to $R_{L_{2}}$. Using a generalization of Kuranishi's construction of the versal deformation space of a compact complex manifold a differential graded Lie algebra (satisfying certain topological hypotheses) determines an analytic germ $\mathscr{K}_{L}$ (the "Kuranishi space") for which the completion of its local ring is $R_{L}$. We call such a differential graded Lie algebra an analytic differential graded Lie algebra. In the case considered above the Kuranishi space is identified with the space of solutions of the deformation equation lying in a slice for the action of group of gauge transformations on $\mathscr{A}(P)$. The main point of [8] (see also [9]) is that an algebraic (i.e. not necessarily continuous) homomorphism of analytic differential graded Lie algebras inducing an isomorphism of cohomology induces an analytic equivalence of Kuranishi spaces.

Quadratic singularities arise as follows. Suppose that the differential in a differential graded Lie algebra is identically zero. Then the defining equations (1) for $Y_{L}(A)$ reduce to the homogeneous quadratic equation

$$
[\omega, \omega]=0
$$

and $Y_{L}(A)$ is the set of $A$-points over the origin of the quadratic cone $\mathscr{Q}_{L}$ in $L^{1}$ defined by (2). A differential graded Lie algebra $L$ is said to be formal if there exists a sequence of differential graded Lie algebra homomorphisms

$$
L=L_{0} \rightarrow L_{1} \leftarrow L_{2} \rightarrow \cdots \leftarrow L_{m-1} \rightarrow L_{m},
$$

where $L_{m}$ has zero differential and each homomorphism induces an isomorphism $H\left(L_{i}\right) \cong H\left(L_{i+1}\right)$. Necessarily $L_{m} \cong H(L)$. The invariance of the Kuranishi space $\mathscr{K}_{L}$ under such homomorphisms of $L$ then leads to the following theorem [8, Theorem 5.3]:

0.1 Theorem. Suppose $(L, d)$ is a formal analytic differential graded Lie algebra. Then the Kuranishi space $\mathscr{K}_{L}$ of $L$ is isomorphic to the quadratic cone $\mathscr{Q} \subset H^{1}(L)$ given by

$$
\mathscr{Q}=\left\{\eta \in H^{1}(L):[\eta, \eta]=0\right\} .
$$

In [7] these ideas are used to show that under certain hypotheses (e.g. $G$ compact) that the space of flat connections on a principal $G$-bundle $P$ over a 
compact Kähler manifold has quadratic singularities. The main technique is to decompose the complexified de Rham algebra $\Omega^{*}(M ; \operatorname{ad} P) \otimes \mathbb{C}$ by Hodge type and its differential as

$$
d=\partial+\bar{\partial}
$$

where

$$
\partial: \Omega^{i, j}(M ; \operatorname{ad} P) \otimes \mathbb{C} \rightarrow \Omega^{i+1, j}(M ; \operatorname{ad} P) \otimes \mathbb{C}
$$

and

$$
\bar{\partial}: \Omega^{i, j}(M ; \operatorname{ad} P) \otimes \mathbb{C} \rightarrow \Omega^{i, j+1}(M ; \operatorname{ad} P) \otimes \mathbb{C} .
$$

As in [5], the Kähler identities then imply that the natural maps

$$
\Omega *(M ; \operatorname{ad} P \otimes \mathbb{C}) \hookleftarrow \operatorname{Ker} \partial \rightarrow H^{*}(M ; \operatorname{ad} P) \otimes \mathbb{C}
$$

are homomorphisms which induce isomorphisms of homology.

Thus in the case of flat principal $G$-bundles over a compact Riemann surface, we have two proofs of quadraticity: one proof inspired by the ideas of Arms-Marsden-Moncrief [2], and another proof which used formality of the de Rham algebra as suggested by Deligne [4]. As the latter techniques seem more fundamental it was natural to try to cast the ideas of Arms-Marsden-Moncrief in Deligne's framework. This is the purpose of the present paper.

This paper is organized as follows. In Sect. 1, we introduce a "universal" graded Lie algebra $\left(5\right.$ associated to a point $x_{0}$ on a symplectic affine space $E$. Furthermore (5 admits a natural derivation $d$ of degree 1. (However $d \circ d:\left(5^{0} \rightarrow\left(5^{2}\right.\right.$ is nonzero; indeed it is an isomorphism.) The elements of degree 0 are quadratic functions $E \rightarrow \mathbb{R}$ (modulo constants), or equivalently affine vector fields preserving the symplectic structure: an infinitesimally symplectic affine vector field is the Hamiltonian vector field of a quadratic function which is unique up to constants and thus there is a natural action of $6^{0}$ on $V$ by affine Hamiltonian vector fields. We take $\left(\mathfrak{5}^{1}=V\right.$ (where $V$ is the vector space associated to $E$ ) and $\left(5^{2}\right.$ to be the vector space dual to $\boldsymbol{6 5}^{\circ}$.

In Sect. 2, we consider subalgebras $\mathfrak{a}$ of $\left(5^{0}\right.$ having the property that $d(\mathfrak{a})$ is an isotropic subspace of $V$; taking $L^{0}=\mathfrak{a}, L^{1}=V$ and $L^{2}=\left(L^{0}\right)^{*}$ we construct (from (5 a differential graded Lie algebra $(L, d)$. (Indeed that $d(\mathfrak{a})$ be isotropic is equivalent to having $d \circ d=0$.) We call such differential graded Lie algebras surface-like since we characterize them abstractly by the existence of a duality pairing analogous to Poincaré duality in dimension two. (In the gauge-theoretic application this pairing is precisely Poincaré duality.) In Sect. 2.2 it is shown that the operator $\Phi: L^{1} \rightarrow L^{2}$ defined by

$$
\Phi(v)=d v+\frac{1}{2}[v, v]
$$

is an equivariant momentum map for the affine action $\bar{\alpha}: L^{0} \rightarrow \mathfrak{a f f}\left(L^{1}\right)$ defined by

$$
\bar{\alpha}(f): v \mapsto[f, v]-d f .
$$

In 2.3 it is shown that there is an isomorphism between the categories of affine Hamiltonian actions together with a zero of an equivariant momentum mapping 
and surface-like differential graded Lie algebras. The cohomology of a surface-like differential graded Lie algebra is interpreted in terms of the symplectic geometry in 2.4 .

In Sect. 3, we consider surface-like diferential graded Lie algebras such that the associated affine action preserves a positive definite complex structure compatible with the symplectic structure. The main result (Theorem 3.2) is that such a differential graded Lie algebra is formal in the sense of [7]. The proof mimics the Hodge theory of Kähler manifolds used in $[5,7]$ by decomposing the complexification $L \otimes \mathbb{C}$ by "Hodge bidegree." Formality is proved by the same arguments used in $[5,7]$.

In Sect. 4 we apply the results of [8] to deduce Theorems A and B.

\section{The Graded Lie Algebra Associated to a Symplectic Vector Space}

1.1. An graded Lie algebra is a graded vector space

with a bilinear operation

$$
\mathfrak{G}=\bigoplus_{i \geqq 0} \mathfrak{G}^{i}
$$

$$
[,]: \mathfrak{G}^{i} \times \mathfrak{G}^{j} \rightarrow \mathfrak{G}^{i+j}
$$

which is graded skew-commutative:

$$
[x, y]+(-1)^{i j}[y, x]=0
$$

and satisfies the graded Jacobi identity:

$$
(-1)^{k i}[x,[y, z]]+(-1)^{i j}[y,[z, x]]+(-1)^{j k}[z,[x, y]]=0
$$

for $x \in \mathfrak{G}^{i}, y \in \mathfrak{G}^{j}, z \in \mathfrak{G}^{k}$. A derivation of degree $l$ is a family $d: \mathfrak{G} \rightarrow \mathfrak{G}$ of maps

$$
d^{i}:\left(\mathfrak{b}^{i} \rightarrow \mathfrak{G}^{i+l}\right.
$$

satisfying

$$
d^{i+j}([x, y])=\left[d^{i} x, y\right]+(-1)^{i l}\left[x, d^{j} y\right]
$$

for $x \in\left(\mathfrak{G}^{i}, y \in \mathfrak{G}^{j}\right.$. The graded Jacobi identity is easily seen to be equivalent (assuming graded skew-commutativity) to the condition that for each $x \in \mathfrak{b}^{i}$ the adjoint transformation

$$
\begin{aligned}
\operatorname{ad}(x): \mathfrak{G}^{j} & \rightarrow \mathfrak{G}^{i+j} \\
y & \mapsto[x, y]
\end{aligned}
$$

is a derivation of degree $i$. A differential graded Lie algebra is a graded Lie algebra with a derivation $d$ of degree 1 such that $d \circ d=0$.

1.2. Let $V$ be a real vector space with a symplectic structure $\langle$,$\rangle and let V^{*}$ denote its dual vector space. The symplectic structure defines an isomorphism

$$
\begin{aligned}
\#: V^{*} & \rightarrow V \\
\psi & \mapsto \psi^{\#}
\end{aligned}
$$


by

$$
\left\langle\psi^{\#}, v\right\rangle=\psi(v) .
$$

In infinite dimensions we only require that $V$ have a weak symplectic structure, in which case we replace $V^{*}$ by the subspace $V^{b} \subset V^{*}$, where \# is defined.

1.3. A function $h: V \rightarrow \mathbb{R}$ is homogeneous quadratic if there is a symmetric bilinear form $h: V \times V \rightarrow \mathbb{R}$ such that $h(v)=\frac{1}{2} h(v, v)$; in this way we shall identify homogeneous quadratic functions with symmetric bilinear forms. (In infinite dimensions, we require that a symmetric bilinear form satisfy the condition that for each $v \in V$, the linear functional

$$
l_{v}(h): u \mapsto h(u, v)
$$

lies in $V^{b}$.) We say that a function $f: V \rightarrow \mathbb{R}$ is quadratic if $f=f_{2}+f_{1}+f_{0}$, where $f_{2}$ is homogenous quadratic, $f_{1} \in V^{b}$ is a linear functional and $f_{0} \in \mathbb{R}$ is a constant function. Let $\mathscr{Q}^{\prime}$ denote the vector space of all quadratic functions $V \rightarrow \mathbb{R}$ and let $\mathbb{R} \hookrightarrow \mathscr{Q}^{\prime}$ denote the inclusion of constant functions. Then $\mathscr{2}^{\prime}$ is a Lie algebra under Poisson bracket and the subspace of constants is its center. Let $\mathscr{Q}=\mathscr{Q}^{\prime} / \mathbb{R}$ denote the quotient Lie algebra of quadratic functions modulo constants. As a vector space 2 may be identified with quadratic functions $f: V \rightarrow \mathbb{R}$ satisfying $f(0)=0$. For any $f \in \mathscr{Q}$ there is a decomposition.

$$
f=f_{2}+f_{1},
$$

where $f_{2}$ is homogeneous quadratic and $f_{1} \in V^{b}$ is linear. For any $v \in V$, let $\pi_{v}: V \rightarrow T_{v} V$ be the linear isomorphism determined by parallel translation by $v$; then $f_{1}(v)=d f_{(0)}\left(\pi_{0}(v)\right)$, where $d f_{(0)}: T_{0} V \rightarrow \mathbb{R}$ is the differential of $f$ at $0 \in V$. By polarization $f_{2}$ determines a symmetric bilinear form (also denoted $f_{2}$ ) which is defined by

$$
f_{2}(u, v)=f(u+v)-f(u)-f(v)+f(0)
$$

and for any vector $u \in V$, the linear functional

$$
v \mapsto f_{2}(u, v)
$$

will be denoted $l_{u}\left(f_{2}\right) \in V^{b}$. Clearly $l_{u}\left(f_{2}\right)$ is related to the Hessian of $f_{2}$ at 0 by

$$
\left(d^{2} f_{2}\right)_{(0)}\left(\pi_{0}(u), \pi_{0}(v)\right)=l_{u}\left(f_{2}\right)(v)
$$

for $u, v \in V$ and to the differential of $f_{2}: V \rightarrow \mathbb{R}$ at $u \in V$ by

$$
\left(d f_{2}\right)_{(u)}\left(\pi_{u} v\right)=l_{u}\left(f_{2}\right)(v)
$$

Let $x \in V$ be an arbitrary point and let $\tau_{x}: u \mapsto u+x$ denote translation by $x$. If $f \in \mathscr{Q}^{\prime}$ is a quadratic function, then the composition $f \circ \tau_{x}: V \rightarrow \mathbb{R}$ is also quadratic and translation $\tau_{x}$ changes the decomposition of $f \in \mathscr{Q}^{\prime}$ as follows:

$$
\left(f \circ \tau_{x}\right)_{2}=f_{2}, \quad\left(f \circ \tau_{x}\right)_{1}(u)=f_{1}(u)+f_{2}(x, u) .
$$

Let $\operatorname{aff}(V)$ denote the space of all affine maps $V \rightarrow V$. Regarding affine maps $V \rightarrow V$ as affine vector fields on $V$, there is a natural Lie algebra structure on $\operatorname{aff}(V)$ 
as the Lie algebra of infinitesimal affine transformations of $V$. It is well-known that the map $\alpha: \mathscr{Q} \rightarrow \mathfrak{a f f}(V)$ defined by

$$
\alpha(f): v \mapsto\left(l_{v}\left(f_{2}\right)\right)^{\#}+f_{1}^{\#}
$$

is a Lie algebra homomorphism taking $\mathscr{2}$ isomorphically onto the algebra of all affine vector fields preserving the symplectic structure on $V$. The condition that $\alpha$ is a Lie algebra homomorphism decomposes into two conditions: first, its linear part

$$
\mathbf{L} \alpha(f): v \mapsto\left(l_{v}\left(f_{2}\right)\right)^{\#}
$$

is a Lie algebra homomorphism $\mathscr{Q} \rightarrow \mathfrak{g l}(V)$; second, its translational part

$$
f \mapsto f_{1}^{\#}
$$

is a derivation $d: \mathscr{Q} \rightarrow V$ of the module defined by $\mathbf{L} \alpha: \mathscr{Q} \rightarrow \operatorname{gl}(V)$. Furthermore if $x \in V$, then the corresponding translation $\tau_{x}$ leaves invariant the linear part of $\alpha(f)$ and changes the translational part $\alpha(f)(0)=f_{1}^{\#}$ to

$$
\left(\tau_{x}\right)_{*}(\alpha(f))(0)=f_{1}^{\#}+\left(l_{x} f_{2}\right)^{\#} \text {. }
$$

1.4. We construct a graded Lie algebra $(5$ associated to the symplectic vector space $V$ as follows. Let $\left(5^{\circ}=\mathscr{2}\right.$ with the Lie algebra structure described above. Consider a family of $\mathfrak{5}^{0}$-modules $\mathfrak{G}^{i}$, for $i>0$. Let $\lambda^{i}:\left(\mathfrak{5}^{0} \rightarrow \operatorname{gl}\left(\mathfrak{G}^{i}\right)\right.$ denote the corresponding Lie algebra representations. We define bracket operations $[,]_{0}$ on the direct sum

$$
\mathfrak{G}=\bigoplus_{i \geqq 0} \mathfrak{G}^{i}
$$

extending the Lie algebra structure on $\left(5^{\circ}\right.$ by

$$
[f, \xi]_{0}=\lambda^{i}(f) \xi \text { and }[\xi, f]_{0}=(-1)^{i+1} \lambda^{i}(f) \xi
$$

(where $f \in{\left(\mathfrak{5}^{0}\right.}^{0}, \xi \in\left(\mathfrak{G}^{i}\right.$ for $i>0$ ) such that

$$
[,]_{0}: \mathfrak{G}^{i} \times \boldsymbol{6}^{j} \rightarrow \boldsymbol{6}^{i+j}
$$

is identically zero for $i>0$ and $j>0$. Clearly with this operation $\mathfrak{G}$ is a graded Lie algebra.

In our application we take $\mathfrak{5}^{1}=V$ with the $\left(\mathfrak{5}^{0}\right.$-module structure $\lambda^{1}=\mathbf{L} \circ \alpha: \mathfrak{5}^{0} \rightarrow \mathfrak{g l}(V)$ defined above and $\mathfrak{5}^{2}$ to equal the vector space $2^{*}$ dual to $\mathscr{Q}=\mathfrak{5}^{0}$ with the coadjoint representation $\lambda^{2}=\operatorname{ad}^{*}: \mathscr{Q} \rightarrow \mathfrak{g l}\left(\mathscr{Q}^{*}\right)$ defined by

$$
\operatorname{ad}^{*}(f)(g): \xi \mapsto-\xi([f, g])
$$

for $f, g \in \mathfrak{G}^{0}$ and $\xi \in\left(\mathfrak{G}^{2}=\mathscr{2}^{*}\right.$. We take $\mathfrak{G}^{i}=0$ for $i>2$.

Define a new graded Lie algebra structure on 6 by introducing a twisting cocycle $c: \mathfrak{G}^{1} \times \mathfrak{G}^{1} \rightarrow \mathfrak{G}^{2}$ as follows. Let $u, v, \in \mathfrak{G}^{1}=V$ and $f \in \mathfrak{G}^{0}=\mathscr{Q}$. Let $c(u, v) \in \mathcal{Q}^{*}$ be the linear functional on 2 :

$$
c(u, v): f \mapsto f_{2}(u, v) .
$$

This defines $c: \mathfrak{G}^{1} \times\left(\mathfrak{G}^{1} \rightarrow \mathfrak{G}^{2}\right.$ and decree $c: \mathfrak{5}^{i} \times \mathfrak{G}^{j} \rightarrow \mathfrak{G}^{i+j}$ to be identically zero 
unless $i=j=1$. Define the bracket $[]:,(5 \times \mathbb{5} \rightarrow(5$ by

$$
[x, y]=[x, y]_{0}+c(x, y) \text {. }
$$

(Note that for homogeneous $x$ and $y$, at most one of the summands is nonzero.)

Clearly $c(x, y)=c(y, x)$ and it follows [ , ] is graded skew-commutative. All that remains to be proved is the graded Jacobi identity (1-3). Since $c(x, y)=0$ except if $x, y \in\left(5^{1}\right.$, the graded Jacobi identity for $[,]_{0}$ implies that for $[$,$] except$ in the case that one of $i, j, k$ equals 0 and the other two equal 1 . We may assume $i=0$ and $j=k=1$ and it suffices to prove

$$
[f, c(y, z)]=c([f, y], z)+c(y,[f, z])
$$

for $f \in \mathfrak{G}^{0}=\mathscr{2}, y, z \in \mathfrak{G}^{1}=V$. This being an equation in $\mathfrak{5}^{2}$, we must show that

$$
[f, c(y, z)](g)=c([f, y], z)(g)+c(y,[f, z])(g)
$$

for every $g \in \mathcal{Q}$. Now

$$
[f, c(y, z)](g)=[g, f]_{2}(y, z)=\left[g_{2}, f_{2}\right](y, z)
$$

equals the second directional derivative

$$
y\left(z\left(\left[g_{2}, f_{2}\right]\right)\right)
$$

(where $y, z$ are regarded as tangent vectors) and

$$
c([f, y], z)(g)=g_{2}\left(\left(l_{y} f_{2}\right)^{\#}, z\right) .
$$

Thus (1-5) is reduced to following assertion concerning second derivatives:

1.5. Lemma. Let $a, b: V \rightarrow \mathbb{R}$ be homogeneous quadratic functions and $y, z \in V$. Then

$$
z(y([a, b]))=b\left(\left(l_{y} a\right)^{\#}, z\right)+b\left(\left(l_{z} a\right)^{\#}, y\right) .
$$

Proof. If $f \in C^{\infty}(V)$, denote by $\mathbf{H} f$ its Hamiltonian vector field, defined by

$$
\langle\mathbf{H} f, y\rangle=y f,
$$

where we identify the vector $y \in V$ with the corresponding parallel vector field on $V$. Let $\nabla$ denote the covariant derivative; for any homogeneous quadratic function $\alpha: V \rightarrow \mathbb{R}$ we have:

$$
\nabla_{y} \mathbf{H} a=\mathbf{H}\left(\nabla_{y} a\right)=\mathbf{H}(y a)=\left(l_{y} a\right)^{\#}
$$

(since the symplectic form is parallel the operation $\mathbf{H}$ assigning to a smooth function its Hamiltonian vector field commutes with covariant differentiation).

The Poisson bracket is defined by $[a, b]=\langle\mathbf{H} a, \mathbf{H} b\rangle$ and its directional derivative with respect to the (tangent) vector $y$ is given by the formula $y[a, b]=y\langle\mathbf{H} a, \mathbf{H} b\rangle=\left\langle\nabla_{y}(\mathbf{H} a), \mathbf{H} b\right\rangle+\left\langle\mathbf{H} a, \nabla_{y}(\mathbf{H} b)\right\rangle=\left\langle\left(l_{y} a\right)^{\#}, \mathbf{H} b\right\rangle+\left\langle\mathbf{H} a,\left(i_{y} b\right)^{\#}\right\rangle$, where $\left(l_{y} a\right)^{\#},\left(l_{y} b\right)^{\#}$ denote the corresponding parallel vector fields. Since $\nabla_{z}\left(\left(l_{y} a\right)^{\#}\right)=\nabla_{z}\left(\left(l_{y} b\right)^{\#}\right)=0$, taking a second covariant derivative we obtain 


$$
\begin{aligned}
z y[a, b] & =\left\langle\left(l_{y} a\right)^{\#}, \nabla_{z}(\mathbf{H} b)\right\rangle+\left\langle\nabla_{z}(\mathbf{H} a),\left(l_{y} b\right)^{\#}\right\rangle \\
& =\left\langle\left(l_{y} a\right)^{\#},\left(l_{z} b\right)^{\#}\right\rangle+\left\langle\left(l_{z} a\right)^{\#},\left(l_{y} b\right)^{\#}\right\rangle \\
& =b\left(\left(l_{y} a\right)^{\#}, z\right)+b\left(\left(l_{z} a\right)^{\#}, y\right)
\end{aligned}
$$

as desired.

It follows that $\mathfrak{6}$ is a graded Lie algebra.

1.6. Next we define a derivation $d:(\mathfrak{5} \rightarrow(\mathfrak{G}$ of degree 1 . In a sense later to be made precise, this derivation is "associated" to the origin $0 \in V$. If $f \in\left(\mathfrak{5}^{0}=\mathscr{Q}\right.$, then define

$$
d^{0}(f)=-f_{1}^{\#} \in V=\mathbf{6}^{1}
$$

and if $v \in \mathfrak{G}^{1}=V$, its image $d^{1} v \in\left(\mathfrak{G}^{2}=\mathscr{Q}^{*}\right.$ is defined by

$$
d^{1}(v): f \mapsto f_{1}(v)
$$

To see that $d$ is a derivation, we first check that

Now

$$
d^{0}([f, g])=\left[d^{0} f, g\right]+\left[f, d^{0} g\right] \text {. }
$$

$$
\begin{aligned}
& d^{0}[f, g]=-[f, g]_{1}^{\#}=-\left[f_{1}, g_{2}\right]^{\#}-\left[f_{2}, g_{1}\right]^{\#}=\left(l_{\left(f_{1}^{\#}\right)} g_{2}\right)^{\#}-\left(l_{\left(g_{1}^{\#}\right)} f_{2}\right)^{\#} \\
& =-\left(l_{\left(d^{\circ} f\right)} g_{2}\right)^{\#}+\left(l_{\left(d^{0} g\right)} f_{2}\right)^{\#}=\left[d^{0} f, g\right]+\left[f, d^{0} g\right]
\end{aligned}
$$

as desired. It remains to show that if $f, g, \in \mathfrak{G}^{0}, v \in \mathfrak{G}^{1}$, then

$$
d^{1}[f, v](g)=\left[d^{0} f, v\right](g)+\left[f, d^{1} v\right](g) .
$$

To this end we prove the following elementary fact:

1.7. Lemma. If $a_{1}, b_{2}: V \rightarrow \mathbb{R}$ are a linear and homogeneous quadratic function, respectively, and $v \in V$, then the Poisson bracket $\left[a_{1}, b_{2}\right]$ satisfies

$$
\left[a_{1}, b_{2}\right](v)=\left\langle a_{1}^{\#},\left(l_{v} b_{2}\right)^{\#}\right\rangle \text {. }
$$

Proof. As usual we confuse vectors with the corresponding parallel vector fields. Now the Poisson bracket $\left[a_{1}, b_{2}\right]$ is a linear function so its value $\left[a_{1}, b_{2}\right](v)$ at $v \in V$ equals the directional derivative

by $(1-6)$.

$$
\begin{aligned}
v\left[a_{1}, b_{2}\right] & =v\left\langle\mathbf{H} a_{1}, \mathbf{H} b_{2}\right\rangle=v\left\langle a_{1}^{\#}, \mathbf{H} b_{2}\right\rangle \\
& =\left\langle a_{1}^{\#}, \nabla_{v}\left(\mathbf{H} b_{2}\right)\right\rangle=\left\langle a_{2}^{\#},\left(l_{v} b_{2}\right)^{\#}\right\rangle
\end{aligned}
$$

Now

$$
d^{1}[f, v](g)=g_{1}\left(\left[f_{2}, v\right]\right)=g_{1}\left(\left(l_{v} f_{2}\right)^{\#}\right)=\left\langle g_{1}^{\#},\left(l_{v} f_{2}\right)^{\#}\right\rangle=\left[g_{1}, f_{2}\right](v)=\left[f_{2}, g_{1}\right](v)
$$

(by Lemma 1.7) and

$$
\left[d^{0} f, v\right](g)=-\left[f_{1}^{\#}, v\right](g)=-g_{2}\left(f_{1}^{\#}, v\right)=-\left(l_{v} g_{2}\right)\left(f_{1}^{\#}\right)=-\left\langle\left(l_{v} g_{2}\right)^{\#},\left(f_{1}^{\#}\right)\right\rangle=\left[f_{1}, g_{2}\right](v)
$$

(again using Lemma 1.7). But

$$
\left[f, d^{1} v\right](g)=-\left(d^{1} v\right)[f, g]=-[f, g]_{1}(v)=-\left[f_{1}, g_{2}\right](v)-\left[f_{2}, g_{1}\right](v)
$$

from which (1-7) follows. 
1.8. Thus $\mathfrak{6}$ is a graded Lie algebra and $d:(\mathfrak{G} \rightarrow \mathfrak{G}$ is a derivation of degree 1 . However, the composition $d \circ d$ is nonzero: indeed, if $f, g \in \mathcal{Q}$, then we have

$$
d^{1} d^{0} f: g \mapsto\left\langle f_{1}^{\#}, g_{1}^{\#}\right\rangle .
$$

Furthermore we have the following relation between the derivation and the symplectic structure. Let $f \in \mathfrak{G}^{0}, v \in \mathfrak{G}^{1}$; then

$$
\left\langle d^{0} f, v\right\rangle=-d^{1} v(f) \text {. }
$$

Both of these facts follow immediately from the definitions and so their proofs are omitted.

1.9. Now suppose that $E$ is a symplectic affine space, a symplectic manifold upon which a vector space $V$ acts simply transitively preserving the symplectic structure. We call $V=V(E)$ the vector space of translations of $E$ or the underlying vector space of $E$. The simply transitive action then identifies each tangent space $T_{u} E$ with $V$ and hence $V$ is a symplectic vector space. Furthermore translating $u$ defines an identification of $V$ with $E$ and it follows that there is an isomorphism of categories between symplectic vector spaces $V$ and pairs $(E, u)$ where $E$ is a symplectic affine space and $u \in E$.

Let $6(E, u)$ denote the graded Lie algebra associated to the symplectic vector space corresponding to $(E, u)$. Since translation by $x \in V$ does not affect the quadratic term $f_{2}$ of an inhomogeneous quadratic function (by (1-4)) and only the quadratic terms of elements of $\mathscr{2}$ enter into the definition of the bracket operation in $\mathbf{5}$, translation $\tau_{x}$ defines canonical isomorphisms $\left(\mathfrak{5}(E, u) \rightarrow \mathfrak{G}\left(E, \tau_{x}(u)\right)\right.$. We shall henceforth identify these isomorphic graded Lie algebras. Let $d_{(u)}:(\mathfrak{G}(E, u) \rightarrow \mathfrak{G}(E, u)$ denote the corresponding derivations constructed in 1.6. If $x \in V(E)$, then it follows from $(1-4)$ and the definitions of the bracket operations and $d^{1}$ that

$$
d_{\left(\tau_{x} u\right)}=d_{(u)}-\operatorname{ad}(x),
$$

where $\operatorname{ad}(x)$ is the derivation of $\mathfrak{5}(E, u)$ given by $y \mapsto[x, u]$.

The constructions of this section can be discussed intrinsically from this point of view. Let $\tilde{5}^{1}$ be the tangent space $T_{x} E$ and $\tilde{5}^{0}$ be the Lie algebra of affine Hamiltonian vector fields. The bracket $\mathfrak{5}^{0} \times \mathfrak{5}^{0} \rightarrow \mathfrak{5}^{0}$ is just the operation in this Lie algebra and the differential $d^{0}:\left(\mathfrak{5}^{0} \rightarrow\left(\mathfrak{5}^{1}\right.\right.$ is just the evaluation map (at $x \in E$ ) which associates to a vector field in $\left(\mathfrak{5}^{0}\right.$ its value at $x\left(\right.$ a vector in $T_{x} E$ ). The bracket operation $\mathfrak{5}^{0} \times \mathfrak{6 5}^{1} \rightarrow \mathfrak{6}^{1}$ is equivalent to a linear representation $\mathfrak{6}^{0} \rightarrow \operatorname{End}\left(T_{x} E\right)$, which corresponds to taking the linear part of the affine vector field. If $\xi \in \mathbb{5}^{0}$ is an affine Hamiltonian vector field and $v \in T_{x} E$, then the bracket $[\xi, v]$ represents the covariant derivative $\nabla_{v} \xi \in T_{x} E$ : For $x, y \in E$ let $\pi_{x, y}: T_{x} E \rightarrow T_{y} E$ denote parallel translation; then $[\xi, v]$ represents the directional derivative at $x$ of the function

$$
\begin{aligned}
E & \rightarrow T_{x} E \\
y & \mapsto \pi_{y, x} \xi(y)
\end{aligned}
$$

with respect to the tangent vector $v \in T_{x} E$.

We take $\left(5^{2}\right.$ to be the vector space dual to $\mathfrak{5}^{0}$ and the action of $\mathfrak{5}^{0}$ on $\mathfrak{5}^{2}$ to be the coadjoint action. It remains to define the differential $d^{1}:\left(5^{1} \rightarrow\left(\mathfrak{b}^{2}\right.\right.$ and the 
bracket $[]:,\left(\mathfrak{5}^{1} \times \mathfrak{G}^{1} \rightarrow \mathfrak{5}^{2}\right.$. Since $\mathfrak{G}^{2}$ is dual to $\mathfrak{G}^{0}$ it suffices to define, for any $\xi \in\left(5^{0}\right.$, the value $d^{1}(v)(\xi)$ for $v \in\left(5^{1}=T_{x} E\right.$. To this end let $d^{1}(v)(\xi)=\omega_{x}(v, \xi(x))$, where $\omega_{x} \in \Lambda^{2} T_{x}^{*} E$ is the symplectic structure on $T_{x} E$. The bracket of two tangent vectors $u, v \in T_{x} E=\left(5^{1}\right.$ is the element of $\left(5^{2}\right.$ (a linear functional on $\left.G^{0}=2\right)$ which assigns to $\xi \in \mathscr{Q}$ the derivative of the function

$$
f_{x, v, \xi}: y \mapsto \omega_{x}\left(v, \pi_{y, x} \xi(y)\right)
$$

with respect to $u \in T_{x} E$. If $\xi$ is the Hamiltonian vector field with potential function $\phi: E \rightarrow \mathbb{R}$, then $d^{1}(v)(\xi)$ equals the differential $d \phi: T_{x} E \rightarrow T_{\phi(x)} \mathbb{R} \cong \mathbb{R}$ and $[u, v](\xi)$ equals the value $d^{2} \phi(u, v)$ where

$$
\nabla d \phi=d^{2} \phi: T_{x} E \times T_{x} E \rightarrow \mathbb{R}
$$

is the Hessian of $\phi$ at $x$. (Notice that although the (quadratic) function $\phi$ is only determined to an additive constant, both $d \phi$ and $d^{2} \phi$ are unambiguously defined.)

\section{Surface-Like Differential Graded Lie Algebras}

2.1. Let $E$ be a symplectic affine space and $u \in E$ a point. Let $\mathfrak{G}=\mathfrak{G}(E, u)$ and $d=\left(d^{0}, d^{1}\right):(5 \rightarrow(5$ be the corresponding graded Lie algebra with derivation constructed in Sect. 1. Let $a \subset \sigma^{\circ}$ be a Lie subalgebra. We say that $a$ is isotropic if the image $d^{0}(a) \subset V$ is an isotropic subspace of $V$ (i.e. the symplectic form restricts to zero on $\left.d^{0}(\mathfrak{a})\right)$. Let $L^{0}=\mathfrak{a}$, let $L^{1}=V$ as before and let $L^{2}=(\mathfrak{a})^{*}$ be the coadjoint module for $a$. We define the structure of a differential graded Lie algebra on

$$
L=L^{0} \oplus L^{1} \oplus L^{2}
$$

as follows. The bracket operations $L^{i} \times L^{j} \rightarrow L^{i+j}$ for $i+j<2$ are just the restrictions of the bracket operations on $\left(5\right.$. The bracket operation $L^{0} \times L^{2} \rightarrow L^{2}$ is the coadjoint action

and

$$
[f, \psi]=\operatorname{ad}^{*} f(\psi)
$$

$$
[\psi, f]=-[f, \psi]
$$

for $f \in L^{0}, \psi \in L^{2}$. Finally the bracket operation $L^{1} \times L^{1} \rightarrow L^{2}$ is the composition

$$
L^{1} \times L^{1}=\mathfrak{G}^{1} \times \mathfrak{G}^{1} \stackrel{[,]}{\rightarrow}{\left(5^{2}\right.}^{\longrightarrow} \rightarrow L^{2},
$$

where $\Pi:\left(^{2}=\left(5^{0}\right)^{*} \rightarrow\left(L^{0}\right)^{*}=L^{2}\right.$ denotes the transpose of the inclusion $\imath: L^{0} \subset\left(^{0}\right.$. Similarly the differentials $d^{0}: L^{0} \rightarrow L^{1}$ and $d^{1}: L^{1} \rightarrow L^{2}$ are defined to be the compositions

and

$$
d^{0}: L^{0} \stackrel{1}{\hookrightarrow} \mathbb{G}^{0} \stackrel{d^{0}}{\rightarrow} \mathbb{6}^{1}=L^{1}
$$

$$
d^{1}: L^{1}=\mathfrak{G}^{1} \stackrel{d^{1}}{\rightarrow} \mathfrak{G}^{2} \stackrel{\Pi}{\rightarrow} L^{2}
$$

respectively. One checks easily that $L$ so defined is a graded Lie algebra and $d: L \rightarrow L$ is a derivation of degree 1 . Furthermore it follows from (1-8) that $L^{0}$ is 
isotropic if and only if the composition $d^{1} \circ d^{0}=0$. Thus $L$ is a differential graded Lie algebra as claimed. We will sometimes denote the resulting algebra with derivation by $L(\mathfrak{a}, \alpha, u)$.

2.2. Let $L$ be a differential graded Lie algebra as above. Let $\alpha: L^{0} \rightarrow \mathfrak{a f f}(V)$ be the affine representation constructed in 1.3 restricted to $L^{0}$. By construction the image $\alpha\left(L^{0}\right)$ lies in the subalgebra $\operatorname{Ham}(V)$ of vector fields on $V$ preserving the symplectic structure on $V$; indeed any such vector field is the Hamiltonian vector field $H f$ for a smooth function $f \in C^{\infty}(V)$. Thus there is an exact sequence of Lie algebras

$$
0 \rightarrow \mathbb{R} \rightarrow C^{\infty}(V) \stackrel{H}{\rightarrow} \operatorname{Ham}(V) \rightarrow 0
$$

where $\mathbb{R} \rightarrow C^{\infty}(V)$ is the inclusion of constant functions. As defined in [1, Sect. 4.2.1], a momentum mapping for $\alpha$ is a map $\Phi: V \rightarrow\left(L^{0}\right)^{*}$ such that for each $f \in L^{0}$ the function $\Phi_{f}: V \rightarrow \mathbb{R}$ defined by $x \mapsto \Phi(x)(f)$ satisfies $H \Phi_{f}=\alpha(f)$. If $\Phi$ is equivariant with respect to the $L^{0}$-actions given by $\alpha$ on $V$ and the coadjoint action on $\left(L^{0}\right)^{*}$, then $\alpha$ lifts to a Hamiltonian action, i.e. a Lie algebra homomorphism $\phi: L^{0} \rightarrow C^{\infty}(V)$. We now construct an equivariant momentum map $\Phi: L^{1} \rightarrow L^{2}$ for the action of $L^{0}$ on $L^{1}$ under the assumption that $L$ is isotropic.

Proposition. $L\left(a, \alpha, x_{0}\right)$ is a differential graded Lie algebra (i.e. $\left.d^{1} \circ d^{0}=0\right) \Leftrightarrow$ the action $\alpha$ of $L^{0}$ on $L^{1}$ is Hamiltonian and there exists an equivariant momentum mapping $\Phi$ for this action satisfying

$$
\Phi\left(x_{0}\right)=0
$$

In this case $\Phi: L^{1} \rightarrow L^{2}$ is necessarily given by the formula

$$
\Phi(v)=d^{1}(v)+\frac{1}{2}[v, v] .
$$

Proof. We obtain a linear cross-section $\sigma$ to the extension of $a$ induced from that of 2.2 by defining

$$
\sigma(f)=h_{f},
$$

where $h_{f}$ is the inhomogeneous quadratic function on $V$ satisfying

$$
\mathbf{H} h_{f}=\alpha(f), \quad h_{f}\left(x_{0}\right)=0 .
$$

We let $C(f, g)$ be the cocycle for the above central extension of a computed using the previous cross-section. We have

$$
\begin{aligned}
C(f, g) & =h_{[f, g]}\left(x_{0}\right)-\left\{h_{f}, h_{g}\right\}\left(x_{0}\right)=-\left\{h_{f}, h_{g}\right\}\left(x_{0}\right) \\
& =-\left\langle\mathbf{H} h_{f}\left(x_{0}\right), \mathbf{H} h_{g}\left(x_{0}\right)\right\rangle=-\left\langle d^{0} f, d^{0} g\right\rangle .
\end{aligned}
$$

This cocycle is identically zero if and only if $\mathfrak{a}$ is isotropic. If $\mathfrak{a}$ is isotopic, then $C$ vanishes and the function $\Phi: L^{1} \rightarrow L^{2}$ given by

$$
\Phi(v): f \mapsto h_{f}(v)
$$

is an equivariant momentum mapping. But by definition of $d^{1}$ and [,] we have

$$
h_{f}(v)=f_{1}(v)+f_{2}(v)=d^{1}(v)(f)+\frac{1}{2}[v, v](f) .
$$


Conversely assume that $\alpha$ is Hamiltonian and there exists an equivariant momentum mapping $\Phi$ satisfying $\Phi\left(x_{0}\right)=0$. Then there exists a cross-section $\sigma$ of the above extension of $a$. But since $\Phi\left(x_{0}\right)=0$ we have $\sigma(f)\left(x_{0}\right)=0$ and necessarily

$$
\sigma(f)=h_{f}
$$

(since $h_{f}$ is already determined up to an additive constant). As a consequence the cocycle $C$ vanishes and $\Phi$ is given by the formula above.

\subsection{In analogy with 2-dimensional Poincaré duality we propose:}

Definition. A differential graded Lie algebra is surface-like if and only if there exists $\mathbf{P}^{i}: L^{i} \otimes L^{2-i} \rightarrow \mathbb{R}$ satisfying:

1. For each integer $i, \mathbf{P}^{i}$ is a nondegenerate pairing;

2. $\mathbf{P}(a, b)=(-1)^{i j} \mathbf{P}(b, a)$ for $a \in L^{i}, b \in L^{j}$, where $i+j=2$;

3. $\mathbf{P}([a, b], c)=\mathbf{P}(a,[b, c])$ for $a \in L^{i}, b \in L^{j}, c \in L^{k}$, where $i+j+k=2$;

4. $\mathbf{P}\left(d^{0} a, b\right)+\mathbf{P}\left(a, d^{1} b\right)=0$ for $a \in L^{0}, b \in L^{1}$.

We define a pointed affine Hamiltonian action to be a collection $((\mathfrak{a}, E, \alpha, \Phi), x)$, where $\mathfrak{a}$ is a Lie algebra, $E$ is a symplectic affine space, $\alpha: \mathfrak{a} \rightarrow \mathfrak{a f f}(E)$ is an affine Hamiltonian action with equivariant momentum mapping $\Phi: E \rightarrow \mathfrak{a}^{*}$, and $x \in E$ satisfies $\Phi(x)=0$. A morphism between pointed affine Hamiltonian actions $((\mathfrak{a}, E, \alpha, \Phi), x) \rightarrow\left(\left(\mathfrak{a}^{\prime}, E^{\prime}, \alpha^{\prime}, \Phi^{\prime}\right), x^{\prime}\right)$ consists of an isomorphism $F: \mathfrak{a} \rightarrow \mathfrak{a}^{\prime}$ of Lie algebras and an isomorphism $f: E \rightarrow E^{\prime}$ of symplectic affine spaces such that

$$
f_{*} \circ \alpha=\alpha^{\prime} \circ F, \quad F^{*} \circ \Phi^{\prime} \circ f=\Phi, \quad f(x)=x^{\prime} .
$$

Theorem. The constructions above define isomorphisms between the category of pointed affine Hamiltonian actions and the category of surface-like differential graded Lie algebras with morphisms differential graded Lie algebra homomorphisms preserving $\mathbf{P}$.

Proof. Suppose that $\psi: L \rightarrow L^{\prime}$ is a homomorphism of surface-like differential graded Lie algebras which preserves the duality pairings $\mathbf{P}, \mathbf{P}^{\prime}$ :

$$
\mathbf{P}^{\prime}(\psi(a), \psi(b))=\mathbf{P}(a, b)
$$

for $a, b \in L$. Since $\mathbf{P}, \mathbf{P}^{\prime}$ are nondegenerate, $\psi$ is an isomorphism of differential graded Lie algebras. It follows that $\psi$ defines an isomorphism $L^{0} \rightarrow L^{\prime 0}$ of Lie algebras and an isomorphism $\left(L^{1}, \mathbf{P}\right) \rightarrow\left(L^{\prime 1}, \mathbf{P}^{\prime}\right)$ of symplectic vector spaces. Taking $\mathfrak{a}=L^{0}, E$ the symplectic affine space corresponding to $\left(L^{\prime}, \mathbf{P}\right)$,

$$
\alpha(X)(u)=[X, u]-d^{0}(X), \quad \Phi(u)=d^{1}(u)+\frac{1}{2}[u, u],
$$

and $x$ the origin in $L^{1}$, etc., we obtain pointed affine Hamiltonian actions with an isomorphism between them. Conversely if

$$
((\mathfrak{a}, E, \alpha, \Phi), x) \rightarrow\left(\left(\mathfrak{a}^{\prime}, E^{\prime}, a^{\prime}, \Phi^{\prime}\right), x^{\prime}\right)
$$

is an isomorphism of pointed affine Hamiltonian actions, then define $L^{0}=\mathfrak{a}$, $L^{1}=T_{x} E$ (the symplectic vector space underlying $E$ ), $L^{2}=\mathfrak{a}^{*}$ with differentials given by

$$
d^{0}(\lambda)=\alpha(\lambda)(0), \quad d^{1}(u): \lambda \mapsto D \Phi_{\lambda}(u)
$$


for $\lambda \in L^{0}, u \in L^{1}$. (Here $\Phi_{\lambda}$ defines the map $E \rightarrow \mathbb{R}$ defined by $\Phi_{\lambda}(u)=\Phi(u)(\lambda)$ and $D \Phi_{\lambda}: T_{x} E \rightarrow \mathbb{R}$ its differential.) The bracket operations for $L^{0}$ are given by the adjoint action, the linear part of $\alpha$, and the coadjoint action (on $L^{0}, L^{1}$, and $L^{2}$ respectively) and for $u, v \in L^{1}$

$$
[u, v](\lambda)=D^{2} \Phi_{\lambda}(u, v),
$$

where $D^{2} \Phi_{\lambda}: T_{x} E \times T_{x} E \rightarrow \mathbb{R}$ is the Hessian of $\Phi_{\lambda}$. The duality operator $\mathbf{P}$ is the given duality between $L^{0}$ and $L^{2}$ and the given symplectic structure on $L^{1}$. Clearly an isomorphism of pointed affine Hamiltonian actions defines an isomorphism of surface-like differential graded Lie algebras.

2.4. The cohomology of $L$ can be interpreted in terms of symplectic geometry as follows. $H^{0}(L)=\operatorname{Ker} d^{0}: L^{0} \rightarrow L^{1}$ is the isotropy Lie algebra of $0 \in V$ and $H^{2}(L)=$ Coker $d^{1}: L^{1} \rightarrow L^{2}$ is dually paired to the isotropy algebra $H^{0}(L)$ (because of (1-9)). The evaluation map $A \rightarrow L^{1}$ at the orbit of 0 has differential equal to $d^{0}: L^{0} \rightarrow L^{1}$ and thus the tangent space to the orbit $A(0)$ equals $B^{1}(L)=$ image $d^{0}$. The differential of the momentum mapping $\Phi: L^{1} \rightarrow L^{2}$ at $x$ equals $d^{1}: L^{1} \rightarrow L^{2}$ and thus the Zariski tangent space to $\Phi^{-1}(0)$ at $x$ equals $Z^{1}(L)=\operatorname{Ker} d^{1}$. It follows that $H^{1}(L)$ represents the normal space to the orbit $A(0)$ inside the level set $\Phi^{-1}(0)$, i.e. the "Zariski tangent space" of the reduced space $\Phi^{-1}(0) / A$. Note that $H^{1}(L)$ inherits a symplectic structure from $L^{1}$ since the null space to $Z^{1}(L)=\operatorname{Ker}\left(d^{1}\right)$ equals $B^{1}(L)=\operatorname{Image}\left(d^{0}\right)$ at 0 .

The space $\Phi^{-1}(0)$ is stratified by conjugacy classes of isotropy groups. In particular the set $\mathscr{N}$ of all points with isotropy group conjugate to the isotropy group at 0 is a smooth manifold (see [2, Sect. 4]). The normal space at 0 to the orbit $A(0)$ of 0 ,

$$
\left.T_{0}(\mathcal{N} / A)\right)=T_{0}(\mathcal{N}) / T_{0}(A(0)) \subset H^{1}(L)
$$

is readily seen to be the set of all $v \in H^{1}(L)$ satisfying $u \cdot v=0$ for all $u \in H^{0}(L)$ under the product $H^{0}(L) \times H^{1}(L) \rightarrow H^{1}(L)$.

\section{Hermitian Functions and Formality}

3.1. A complex structure on a symplectic vector space $(V,\langle\rangle$,$) is a linear$ map $\mathbf{J}: V \rightarrow V$ satisfying

1. $\mathbf{J}^{2}=-I$;

2. $\langle\mathbf{J} u, \mathbf{J} v\rangle=\langle u, v\rangle$ for $u, v \in V$.

It follows that if $\psi \in V^{\mathrm{b}}$ then $\psi \circ \mathbf{J} \in V^{b}$ and

$$
(\psi \circ \mathbf{J})^{\#}=-\mathbf{J}\left(\psi^{\#}\right) .
$$

A complex structure $\mathbf{J}: V \rightarrow V$ is positive definite if the symmetric bilinear form defined by

$$
(u, v)=\langle u, \mathbf{J} v\rangle
$$

is positive definite. We will frequently use the following elementary lemma. 
3.2. Lemma. Let $U \subset V$ be a finite dimensional subspace and let $U^{\perp}$ be the orthogonal complement of $U$ with respect to (,). Then $V$ is a direct sum

$$
V=U+U^{\perp} \text {. }
$$

Proof. Let $v \in V$. Since the restriction of (,) to $U$ is positive definite and $U$ is finite dimensional there exists $u \in U$ such that the linear forms

$$
x \rightarrow(x, v) \text { and } x \rightarrow(x, u)
$$

agree on $U$. Hence $w=v-u \in U^{\perp}$ and $v=u+w$.

We say that $f \in \mathscr{Q}$ is Hermitian (with respect to $\mathbf{J}$ ) if for each $v \in V$,

$$
f_{2}(\mathbf{J} v)=f_{2}(v)
$$

or equivalently in polarized form

$$
f_{2}(\mathbf{J} u, \mathbf{J} v)=f_{2}(u, v) .
$$

If $f \in \mathscr{Q}$ is Hermitian and $v \in V$, then

$$
\left(l_{v} f_{2}\right) \circ \mathbf{J}=-l_{(\mathbf{J} v)} f_{2} .
$$

One can check that the Hamiltonian vector field $\mathbf{H} f$ preserves the complex structure $\mathbf{J}$ if and only if $f$ is Hermitian.

3.3. Suppose that $L$ is a surface-like differential graded Lie algebra. We say that $L$ is Hermitian if there is a positive definite complex structure $\mathbf{J}$ on $L^{1}$ such that each $f \in L^{0}$ is Hermitian with respect to $\mathbf{J}$. Our main result is the following:

Theorem. A Hermitian isotropic differential graded Lie algebra is formal.

3.4. Let $L \otimes \mathbb{C}$ be the complexification of $L$ with the bracket operation, the differential and the complex structure extended $\mathbb{C}$-linearly. Then $L \otimes \mathbb{C}$ is a complex differential graded Lie algebra. (Complex conjugation $L \otimes \mathbb{C} \rightarrow L \otimes \mathbb{C}$ is defined and its fixed point set equals $L=L \otimes \mathbb{R} \subset L \otimes \mathbb{C}$.) Similarly extend the symplectic structure $\langle$,$\rangle on L^{1} \otimes \mathbb{C} \mathbb{C}$-bilinearly so the operator $\#:\left(L^{1} \otimes \mathbb{C}\right)^{b} \rightarrow L^{1} \otimes \mathbb{C}$ corresponding to $\langle$,$\rangle is \mathbb{C}$-linear. The complex structure $\mathbf{J}: L^{1} \rightarrow L^{1}$ extends to a $\mathbb{C}$-linear map $L^{1} \otimes \mathbb{C} \rightarrow L^{1} \otimes \mathbb{C}$ (so $\mathbf{J}$ commutes with complex conjugation). On the other hand we extend the bilinear form (,) on $L^{1}$ to a Hermitian form on $L^{1} \otimes \mathbb{C}$ by:

$$
(u, v)=\langle u, \mathbf{J} \bar{v}\rangle .
$$

This Hermitian form on $L^{1} \otimes \mathbb{C}$ induces a positive definite Hermitian form on the vector space $\mathscr{Q} \otimes \mathbb{C} \cong S^{2}\left(L^{1} \otimes \mathbb{C}\right)^{*} \oplus\left(L^{1} \otimes \mathbb{C}\right)^{*}$ of quadratic functions modulo constants, and hence $L^{0} \subset \mathscr{Q} \otimes \mathbb{C}$ inherits a positive definite Hermitian form, which is invariant under $\operatorname{Ad}\left(H^{0}\right)$.

We define

$$
\begin{array}{ll}
L^{0,0}=L^{0} \otimes \mathbb{C}, & L^{1,1}=L^{2} \otimes \mathbb{C}, \\
L^{1,0}=\left\{u \in L^{1} \otimes \mathbb{C} \mid \mathbf{J} u=i u\right\}, & L^{0,1}=\left\{u \in L^{1} \otimes \mathbb{C} \mid \mathbf{J} u=-i u\right\} .
\end{array}
$$

Lemma. $L \otimes \mathbb{C}$ is a bigraded Lie algebra, i.e. $\left[L^{i, j}, L^{i^{\prime}, j^{\prime}}\right] \subset L^{i+i^{\prime}, j+j^{\prime}}$. 
Proof. It suffices to show that $\operatorname{ad}\left(L^{0,0}\right)$ preserves the decomposition

and that

$$
L^{1} \otimes \mathbb{C}=L^{1,0} \oplus L^{0,1}
$$

$$
\left[L^{0,1}, L^{0,1}\right]=\left[L^{1,0}, L^{1,0}\right]=0 .
$$

Suppose that $f \in L^{0,0}$ and $v \in L^{1,0}$. Then $[f, v]=\left(l_{v} f_{2}\right)^{\#}$ and

$$
\mathbf{J}[f, v]=\mathbf{J}\left(l_{v} f_{2}\right)^{\#}=-\left(l_{v} f_{2} \circ \mathbf{J}\right)^{\#}=-\left(-l_{(\mathbf{J v})} f_{2}\right)^{\#}=i\left(l_{v} f_{2}\right)^{\#}=i[f, v],
$$

whence $[f, v] \in L^{1,0}$. Similarly $\left[L^{0,0}, L^{0,1}\right] \subset L^{0,1}$. Thus $\operatorname{ad}\left(L^{0,0}\right)$ preserves the decomposition $L^{1} \otimes \mathbb{C}=L^{1,0} \oplus L^{0,1}$.

To prove $\left[L^{0,1}, L^{1,0}\right]=0$, consider $u, v \in L^{1,0}$ and $f \in \mathscr{Q}$. Then

$$
[u, v](f)=f_{2}(u, v)=f_{2}(\mathbf{J} u, \mathbf{J} v)=f_{2}(i u, i v)=-f_{2}(u, v)=-[u, v](f),
$$

proving $[u, v]=-[u, v]$; therefore $[u, v]=0$ as claimed. Similarly $\left[L^{0,1}, L^{0,1}\right]=0$.

3.5. Next we decompose the differential $d=\partial+\bar{\partial}$. Let

$$
\begin{aligned}
& \pi^{0,1}: L^{1} \otimes \mathbb{C} \rightarrow L^{0,1}, \\
& \pi^{1,0}: L^{1} \otimes \mathbb{C} \rightarrow L^{1,0}
\end{aligned}
$$

denote the projections. Define

$$
\begin{aligned}
& \partial=\pi^{1,0} \circ d^{0}: L^{0,0} \rightarrow L^{1,0} \subset L^{1} \otimes \mathbb{C}, \\
& \bar{\partial}=\pi^{0,1} \circ d^{0}: L^{0,0} \rightarrow L^{0,1} \subset L^{1} \otimes \mathbb{C},
\end{aligned}
$$

and define $\partial, \bar{\partial}: L^{1} \otimes \mathbb{C} \rightarrow L^{1,1}$ by

$$
\begin{aligned}
& \partial: d^{1} \circ \pi^{0,1}: L^{1} \otimes \mathbb{C} \rightarrow L^{1,1} \\
& \bar{\partial}: d^{1} \circ \pi^{1,0}: L^{1} \otimes \mathbb{C} \rightarrow L^{1,1} .
\end{aligned}
$$

Let

$$
B^{1}=\text { Image }\left(d^{0}: L^{0} \otimes \mathbb{C} \rightarrow L^{1} \otimes \mathbb{C}\right),
$$

and let $C^{1}=\left(B^{1}\right)^{\perp}$ be its orthogonal complement in $L^{1} \otimes \mathbb{C}$ with respect to (,).

Lemma. Let $f \in L^{0,0}$ and suppose that either $\partial f=0$ or $\bar{\partial} f=0$. Then $d f=0$.

Proof. Let $g \in L^{0}$. Then $\langle d g, \mathbf{J} d f\rangle= \pm i\langle d g, d f\rangle=0$ (since $B^{1}$ is isotropic with respect to $\langle\rangle$,$) . Thus \mathbf{J} d f \in C^{1}$ and consequently $\mathbf{J} d f \in \mathbf{J} B^{1} \cap C^{1}=B^{1} \cap C^{1}=0$.

3.6. Let $H^{0,0}=\operatorname{Ker}\left(d^{0}: L^{0,0} \rightarrow L^{1} \otimes \mathbb{C}\right)$ and $Z^{1}=\operatorname{Ker}\left(d^{1}: L^{1} \otimes \mathbb{C} \rightarrow L^{2} \otimes \mathbb{C}\right)$. Let $B^{0,0}=\left(H^{0,0}\right)^{\perp}$, whence $L^{0,0}=H^{0,0}+B^{0,0}$ by Lemma 3.2 and let

$$
\mathscr{H}^{1}=Z^{1} \cap \mathbf{J} Z^{1} \text {. }
$$

We define $H^{1,0}=\mathscr{H}^{1} \cap L^{1,0}$ and $H^{0,1}=\mathscr{H}^{1} \cap L^{0,1}$ and obtain

$$
\mathscr{H}^{1}=H^{1,0}+H^{0,1} \text {. }
$$

It is immediate that

$$
H^{1,0}=Z^{1} \cap L^{1,0}, \quad H^{0,1}=Z^{1} \cap L^{0,1},
$$


and we define

$$
\begin{aligned}
& B^{1,0}=\pi^{1,0}\left(B^{1}\right) \subset L^{1,0} \\
& B^{0,1}=\pi^{0,1}\left(B^{1}\right) \subset L^{0,1} .
\end{aligned}
$$

(Note that $B^{1,0}$ and $B^{0,1}$ are not contained in $B^{1}$.)

Proposition. There are orthogonal direct sum decompositions

$$
\begin{aligned}
Z^{1} & =B^{1} \oplus H^{1,0} \oplus H^{0,1}, \\
L^{1,0} & =B^{1,0} \oplus H^{1,0}, \\
L^{0,1} & =B^{0,1} \oplus H^{0,1} .
\end{aligned}
$$

In the following lemma, $\perp$ denotes orthogonal complement with respect to the Hermitian form (,) on $L^{1} \otimes \mathbb{C}$.

\subsection{Lemma.}

(i) $\left(B^{0,1}\right)^{\perp} \cap L^{1,0}=H^{1,0}$,

(ii) $\left(B^{0,1}\right)^{\perp} \cap L^{0,1}=H^{0,1}$.

Proof. We prove (i). Suppose $u \in L^{1,0}$. Then

$$
\begin{aligned}
u \perp B^{1,0} & \Leftrightarrow 0=(\partial f, u)=\left\langle\pi^{1,0} d f, \mathbf{J} \bar{u}\right\rangle \\
& =-i\left\langle\pi^{1,0} d f, \bar{u}\right\rangle=-i\left\langle d f, \pi^{0,1} \bar{u}\right\rangle \\
& =-i\langle d f, \bar{u}\rangle=i\langle f, d \bar{u}\rangle \text { for all } f \in L^{0,0} \\
& \Leftrightarrow d \bar{u}=0 \\
& \Leftrightarrow u \in Z^{1} \\
& \Leftrightarrow u \in Z^{1} \cap L^{1,0}=H^{1,0} .
\end{aligned}
$$

(ii) is proved similarly.

In finite dimensions

$$
L^{1}=B^{1}+C^{1}
$$

and in infinite dimensions we assume $\left({ }^{*}\right)$.

We define $d^{c}: L^{1} \otimes \mathbb{C} \rightarrow L^{1} \otimes \mathbb{C}$ by $d^{c}=\mathbf{J}^{-1} d \mathbf{J}=-i(\partial-\bar{\partial})$

\subsection{Lemma.}

$$
\operatorname{Ker} d^{c}=\left(B^{1}\right)^{\perp}
$$

Proof. Let $a \in L^{0}, b \in L^{1}$. Then

$$
\begin{aligned}
(d a, b) & =\mathbf{P}(d a, \mathbf{J} b)=-\mathbf{P}(a, d \mathbf{J} b) \\
& =-\mathbf{P}\left(a, \mathbf{J}^{-1} d \mathbf{J} b\right)=-\left(a, d^{c} b\right) .
\end{aligned}
$$

Corrollary. $\left(B^{1}\right)^{\perp} \cap Z^{1}=\operatorname{Ker} d^{c} \cap \operatorname{Ker} d=\mathscr{H}^{1}$.

We prove Proposition 3.6. Since $B^{1} \subset Z^{1}$ it follows from $\left(^{*}\right)$ that

$$
\begin{aligned}
Z^{1} & =B^{1}+\left(B^{1}\right)^{\perp} \cap Z^{1}=B^{1}+\mathscr{H}^{1} \\
& =B^{1}+H^{1,0}+H^{0,1}
\end{aligned}
$$


and Lemma 3.2 implies

$$
\begin{aligned}
& L^{1,0}=H^{1,0}+\left(H^{1,0}\right)^{\perp} \cap L^{1,0}=H^{1,0}+B^{1,0}, \\
& L^{0,1}=H^{0,1}+\left(H^{0,1}\right)^{\perp} \cap L^{0,1}=H^{0,1}+B^{0,1} .
\end{aligned}
$$

3.9. We use the above decompositions to split the differential graded Lie algebra $L \otimes \mathbb{C}$ as follows. Decompose

$$
\begin{aligned}
& L^{0,0}=H^{0,0}+B^{0,0}, \\
& L^{1,0}=H^{1,0}+B^{1,0}, \\
& L^{0,1}=H^{0,1}+B^{0,1},
\end{aligned}
$$

and decompose $L^{1,1}$ by defining (here $\perp$ means the orthogonal complement for the pairing $\mathbf{P}$ )

$$
\begin{aligned}
& B^{1,1}=\text { Image }\left(d^{1}\right)=\text { Image } \partial=\text { Image } \bar{\partial}=\left(H^{0,0}\right)^{\perp}, \\
& H^{1,1}=\left(B^{0,0}\right)^{\perp} .
\end{aligned}
$$

Thus $L^{1,1}=H^{1,1}+B^{1,1}$. Observe that $H^{0,0}$ is dually paired with $H^{1,1}$ and that $B^{0,0}$ is dually paired with $B^{1,1}$ and the maps $\partial: B^{0,1} \rightarrow B^{1,1}$ and $\bar{\partial}: B^{1,0} \rightarrow B^{1,1}$ are dual to $\partial: B^{0,0} \rightarrow B^{1,0}$ and $\bar{\partial}: B^{0,0} \rightarrow B^{0,1}$ respectively. Therefore $L \otimes \mathbb{C}$ is a direct sum of the complexes

and the square

$$
H(L)=H^{0,0} \oplus H^{1,0} \oplus H^{0,1} \oplus H^{1,1},
$$

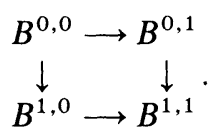

The following lemma is a consequence of the preceding observations.

Lemma. The restrictions of $\partial$

$$
\partial: B^{0,0} \rightarrow B^{1,0}, \quad \partial: B^{0,1} \rightarrow B^{1,1}
$$

and the restrictions of $\bar{\partial}$

$$
\bar{\partial}: B^{0,0} \rightarrow B^{0,1}, \quad \bar{\partial}: B^{1,0} \rightarrow B^{1,1}
$$

are isomorphisms.

3.10. Corollary. $H(L)$ is isomorphic to the cohomology algebra of $L \otimes \mathbb{C}$.

Proof of Theorem 3.2. We define a subalgebra $K \subset L$ by

$$
K=\operatorname{Ker} \partial: L \otimes \mathbb{C} \rightarrow L \otimes \mathbb{C}, \quad K^{0}=H^{0,0}, \quad K^{1}=H^{0,1}+L^{1,0}, \quad K^{2}=L^{1,1},
$$

and $K$ is a differential graded subalgebra of $L$. Furthermore the only nonzero differential is $\bar{\partial}: L^{1,0} \rightarrow L^{1,1}$ and the inclusion $K \subset L$ induces an isomorphism of homology. Now $B^{1,0} \oplus B^{1,1}$ clearly forms an ideal in $K$. Let $H$ denote the quotient algebra; explicitly

$$
H^{0}=H^{0,0}, \quad H^{1}=H^{1,0} \oplus H^{0,1}, \quad H^{2}=H^{1,1},
$$


and the quotient homomorphism $K \rightarrow H$ is orthogonal projection. Furthermore the quotient homomorphism induces isomorphism of cohomology. The proof of Theorem 3.3 is now complete.

\section{The Proofs of Theorems $A$ and $B$}

4.1. Let $Y_{L} \subset L^{1}$ be the Banach analytic set defined by

$$
Y_{L}=\left\{\eta \in C^{1}: d \eta+\frac{1}{2}[\eta, \eta]=0\right\} \text {. }
$$

Thus in terms of symplectic geometry

$$
Y_{L}=\Phi^{-1}(0) \cap S_{x}
$$

The following lemma is clear.

Lemma. The map $\mu: \mathscr{U}_{e} \times S_{x} \rightarrow E$ (see the remarks following Theorem $B$ ) induces $a$ Banach analytic isomorphism of germs

$$
\left(B^{1}, 0\right) \times\left(Y_{L}, 0\right) \rightarrow\left(\Phi^{-1}(0), x\right) .
$$

4.2. We now show that $\left(Y_{L}, 0\right)$ is isomorphic to a finite dimensional germ by using the Kuranishi construction. To apply this construction it suffices to split the short exact sequences

$$
0 \rightarrow Z^{j}(L) \rightarrow L^{j} \stackrel{d}{\rightarrow} B^{j+1}(L) \rightarrow 0
$$

and

$$
0 \rightarrow B^{j}(L) \rightarrow Z^{j}(L) \rightarrow H^{j}(L) \rightarrow 0
$$

for $j=0,1,2$ (see [8, Sect. 2]- since $L$ is already complete the treatment there can be simplified considerably).

We assumed (formula $(*)$ of the introduction) that

$$
L^{1}=B^{1}+C^{1} \text {. }
$$

Let $\mathscr{A}^{1}=\left(\mathscr{H}^{1}\right)^{\perp} \cap C^{1}$. By Lemma 3.2

and

$$
C^{1}=\mathscr{H}^{1}+\mathscr{A}^{1}
$$

$$
L^{1}=B^{1}+\mathscr{H}^{1}+\mathscr{A}^{1} .
$$

Thus we obtain the required splittings in case $j=1$. Now

$$
L^{0}=H^{0,0}+B^{0,0}
$$

and we have constructed the required splittings for the case $j=0$. By duality we obtain splittings for $j=2$.

We can now apply the Kuranishi construction ([8, Sect. 2]). We obtain a finitedimensional analytic germ $\left(\mathscr{K}_{L}, 0\right)$ embedded in $\mathscr{H}^{1}$ such that $\left(Y_{L}, 0\right)$ and $\left.\mathscr{K}_{L}, 0\right)$ are Banach analytically isomorphic. By Theorem 3.3, $L$ is formal and by Theorem 0.1 the finite dimensional germs $\left(\mathscr{K}_{L}, 0\right)$ and $(\mathscr{2}, 0)$ are analytically equivalent. Theorems A and B follow. 


\section{References}

1. Abraham, R., Marsden, J.: Foundations of mechanics, second ed. Reading, MA: Addison Wesley 1978

2. Arms, J., Marsden, J., Moncrief, V.: Symmetry and bifurcation of momentum mappings. Commun. Math. Phys. 78, 455-478 (1981)

3. Atiyah, M., Bott, R.: The Yang-Mills equations over a compact Riemann surface. Phil. Trans. R. Soc. London A308, 523-615 (1982)

4. Deligne, P.: Letter to J. Millson and W. Goldman, April 24, 1986

5. Deligne, P., Griffiths, P. A., Morgan, J. W., Sullivan, D.: Rational homotopy type of compact Kähler manifolds. Invent. Math. 29, 245-274 (1975)

6. Goldman, W. M., Millson, J. J.: Deformations of flat bundles over Kähler manifolds. In: Geometry and Topology, Manifolds, Varieties and Knots. McCrory, C., Shifrin, T. (eds.). Lecture Notes in Pure and Applied Mathematics vol. 105, pp. 129-145. New York, Basel: Marcel Dekker (1987)

7. Goldman, W. M., Millson, J. J.: The deformation theory of representations of fundamental groups of compact Kähler manifolds. Publ. Math. I.H.E.S. 67, 43-96 (1988)

8. Goldman, W. M., Millson, J. J.: The homotopy invariance of the Kuranishi space. Ill. J. Math. (memorial issue dedicated to $\mathrm{K}$. T. Chen)

9. Schlessinger, M., Stasheff, J.: Deformation theory and rational homotopy type. Publ. Math. I.H.E.S. (to appear)

Communicated by J. N. Mather

Received February 26, 1988; in revised form December 21, 1989 
